\title{
Comparative safety and efficacy of anti-PD- 1 monotherapy, chemotherapy alone, and their combination therapy in advanced nasopharyngeal carcinoma: findings from recent advances in landmark trials
}

\author{
Jia-Wei Lv ${ }^{1 \dagger}$, Jun-Yan Li ${ }^{1+}$, Lin-Na Luo ${ }^{2 \dagger}$, Zi-Xian Wang ${ }^{2^{*}}$ (D) and Yu-Pei Chen ${ }^{1 *}$
}

\begin{abstract}
Recent phase 1-2 trials reported manageable safety profiles and promising antitumor activities of anti-PD-1 drugs (pembrolizumab, nivolumab, camrelizumab and JS001) with/without chemotherapy in recurrent/metastatic nasopharyngeal carcinoma (RM-NPC), however head-to-head comparison among these regimens is lacking. We aimed to comprehensively compare the efficacy and safety of different anti-PD-1 drugs, standard chemotherapy, and their combination therapy in RM-NPC. Adverse event (AE) and objective response rate (ORR) were assessed. The pooled incidence rates of grade 1-5/3-5 AEs were 74.1\%/29.6, 54.2\%/17.4, 92.3\%/24.5, 96.8\%/16.1, 91.2\%/42.8, and $100 \% / 87.9 \%$ for pembrolizumab, nivolumab, JS001, camrelizumab, chemotherapy and camrelizumab+chemotherapy, respectively, which suggested that nivolumab and pembrolizumab exhibited the optimal safety regarding grade 1-5 AEs whereas camrelizumab and nivolumab regarding grade 3-5 AEs. As second- or later-line therapy, ORR was higher with camrelizumab (34.1\%), followed by pembrolizumab (26.3\%), JSO01 (23.3\%), and nivolumab (19.0\%); whereas ORR with first-line nivolumab reached 40\%. Additionally, first-line camrelizumab+chemotherapy achieved a dramatically higher ORR than that with chemotherapy alone ( $90.9 \%$ vs. 64.1\%). Pooled ORR was 28.4 and $17.4 \%$ for PD-L1-positive and PD-L1-negative patients, respectively $(P=0.11)$. Here, we represent preliminary evidence for the comparative safety and efficacy of existing anti-PD-1 agents with/ without chemotherapy in RM-NPC, which indicated that camrelizumab has the least toxicity profile and merits future investigation. Our findings might provide insights into the future design of immunotherapy trials in RM-NPC.
\end{abstract}

Keywords: Nasopharyngeal carcinoma, Anti-PD-1, Chemotherapy, Combination therapy, Safety profiles, Efficacy, Predictive biomarker

\footnotetext{
* Correspondence: wangzx@sysucc.org.cn; chenyup1@sysucc.org.cn

${ }^{\dagger} J i a-W e i$ LV, Jun-Yan Li and Lin-Na Luo contributed equally to this work. ${ }^{2}$ Department of Medical Oncology, Sun Yat-sen University Cancer Center, State Key Laboratory of Oncology in South China, Collaborative Innovation Center for Cancer Medicine, Guangzhou, People's Republic of China 'Department of Radiation Oncology, Sun Yat-sen University Cancer Center, State Key Laboratory of Oncology in South China, Collaborative Innovation Center for Cancer Medicine, Guangdong Key Laboratory of Nasopharyngeal Carcinoma Diagnosis and Therapy, 651 Dongfeng Road East, Guangzhou 510060, People's Republic of China
}

(c) The Author(s). 2019 Open Access This article is distributed under the terms of the Creative Commons Attribution 4.0 International License (http://creativecommons.org/licenses/by/4.0/), which permits unrestricted use, distribution, and reproduction in any medium, provided you give appropriate credit to the original author(s) and the source, provide a link to the Creative Commons license, and indicate if changes were made. The Creative Commons Public Domain Dedication waiver (http://creativecommons.org/publicdomain/zero/1.0/) applies to the data made available in this article, unless otherwise stated. 


\section{Background}

Nasopharyngeal carcinoma (NPC) is one of the most common head and neck cancers in Southeast Asia and North Africa. The age-standardized incidence ranges from 20 to 50 per 100,000 males in southern China to 0.5 per 100,000 in white populations [1]. Recently, the first phase 3 trial in recurrent or metastatic NPC (RMNPC), the landmark GEM20110714 study, has established gemcitabine plus cisplatin (GP) regimen as the standard first-line treatment [2]. However, no consensus has been reached beyond the first-line setting, in which the prognosis is extremely poor.

Endemic NPC is etiologically associated with EpsteinBarr virus infection. This virus-associated cancer represents the archetypal "inflamed tumor," which exhibits a dense lymphocytic infiltrate and increased programmed death-ligand 1 (PD-L1) expression [3, 4]. These features make immunotherapy a promising treatment option for NPC patients. Recently in 2017, the landmark KEYNOTE-028 trial firstly reported promising antitumor activities and safety profiles of pembrolizumab in previously treated RM-NPC [5]. Subsequently, five additional phase 1-2 trials evaluating anti-PD-1 antibodies in RM-NPC were reported [6-9]. The NCI-9742 [6] and CheckMate-385 [7] trials demonstrated a manageable safety profile and clinical activity of nivolumab in multiply pretreated and/or treatment-naive RM-NPC patients. Fang and colleagues [8] reported that camrelizumab monotherapy was a well-tolerated and potentially effective treatment option for previously treated RMNPC. They further reported that the combination of camrelizumab plus chemotherapy of GP regimen has a manageable toxicity profile and promising preliminary antitumor activity in treatment-naive RM-NPC [8]. Another latest trial, the JS001 study, reported in the European Society for Medical Oncology (ESMO) 2018 conference demonstrated the clinical activity of JS001 in multiply pretreated RM-NPC [9]. However, to date there is no head-to-head comparison of different anti-PD-1 drugs, standard first-line GP chemotherapy, and their combination therapy in RM-NPC. Therefore, we initiated this study to comprehensively compare the safety and efficacy of the abovementioned trials, and explore the optimal therapeutic regimens of anti-PD-1 approach in RM-NPC. We hypothesized that the efficacy and safety profiles differed across different anti-PD-1-based regimens.

\section{Methods}

The abovementioned anti-PD-1 trials were included in the analysis with GP arm from GEM20110714 trial as chemotherapy control [2]. The major assessed outcomes were adverse event (AE) and objective response rate (ORR). AE and ORR data were pooled up per regimen and described in percentage. The comparative incidences of $\mathrm{AE}$ between different regimens were evaluated by the odds ratio (OR) and corresponding 95\% confidence interval (CI) using Fisher's exact test. OR > 1 stands for fewer AEs. When AE rate in any comparative arm equaled $100 \%$ or $0 \%$, the Haldane-Anscombe correction was adopted to evaluate OR and its 95\% CI [10]. Given that ORR of PD-1 blockade may differ according to treatment lines (first-line vs. $>1$ st line), we also evaluated the anti-PD-1 drugs per treatment setting and considered them as independent comparative groups when data was available. Given the evidence that high PD-L1 expression tended to be associated with favorable responses to PD-1 blockade in NPC [6], we further evaluated the pooled ORR of anti-PD-1 therapies stratified by PD-L1 positivity. Statistical analyses were performed using $\mathrm{R}$ version 3.5.1 (http://www.r-project.org). A twotailed $P<0.05$ was considered statistically significant.

\section{Results}

\section{Safety profile of different regimens}

Table 1 summarizes the characteristics of included trials. The median sample size for anti-PD-1 monotherapy was 45 (range, 24-143), sample sizes for combination therapy and GP chemotherapy were 23 and 181, respectively. Four of the seven (57.1\%) trials investigated anti-PD-1 therapy in pretreated RM-NPC, $2 / 7(28.5 \%)$ trials investigated treatment-naive RM-NPC, while one trial (CheckMate-385) investigated patients receiving $\leq 2$ prior systemic therapies. Figure 1 shows the comparison of safety profiles of anti-PD-1 monotherapy, chemotherapy alone, and their combination. The pooled incidence rates of grade $1-5 / 3-5$ AEs were $74.1 \% / 29.6,54.2 \% / 17.4$, $92.3 \% / 24.5,96.8 \% / 16.1,91.2 \% / 42.8$, and $100 \% / 87.9 \%$ for pembrolizumab, nivolumab, JS001, camrelizumab, chemotherapy, and camrelizumab+chemotherapy, respectively (Fig. 1a). The incidence rate of grade 1-5 AEs was lowest with nivolumab monotherapy, while grade 3-5 AEs was lowest with single-agent camrelizumab. Treatment-related deaths were reported in patients receiving pembrolizumab (sepsis, $n=1$ ) and nivolumab (pulmonary tuberculosis, $n=1$ ) (Fig. 1a). Treatment discontinuation due to AEs was most commonly recorded in pembrolizumab (18.5\%), followed by camrelizumab+chemotheray (13.0\%) and JS001 (9.8\%), while lowest in camrelizumab (2.2\%) (Fig. 1a). Fisher's exact test indicated a noticeably lower risk of grade 1-5 AEs favoring nivolumab and pembrolizumab over other regimens, while nivolumab and camrelizumab demonstrated superior safety ranking to other regimens for grade 3-5 AEs (Fig. 1b). Generally, risks of grade $1-5$ and 3-5 AEs of anti-PD-1 agents were lower than those of chemotherapy alone, while their combination therapy shared the highest incidence of grade 1-5 and 3-5 AEs (Fig. 1b).

To profile the toxicity spectra of different regimens, we further evaluated the incidence of immune-related 
Lv et al. Journal for ImmunoTherapy of Cancer

(2019) 7:159

Page 3 of 9

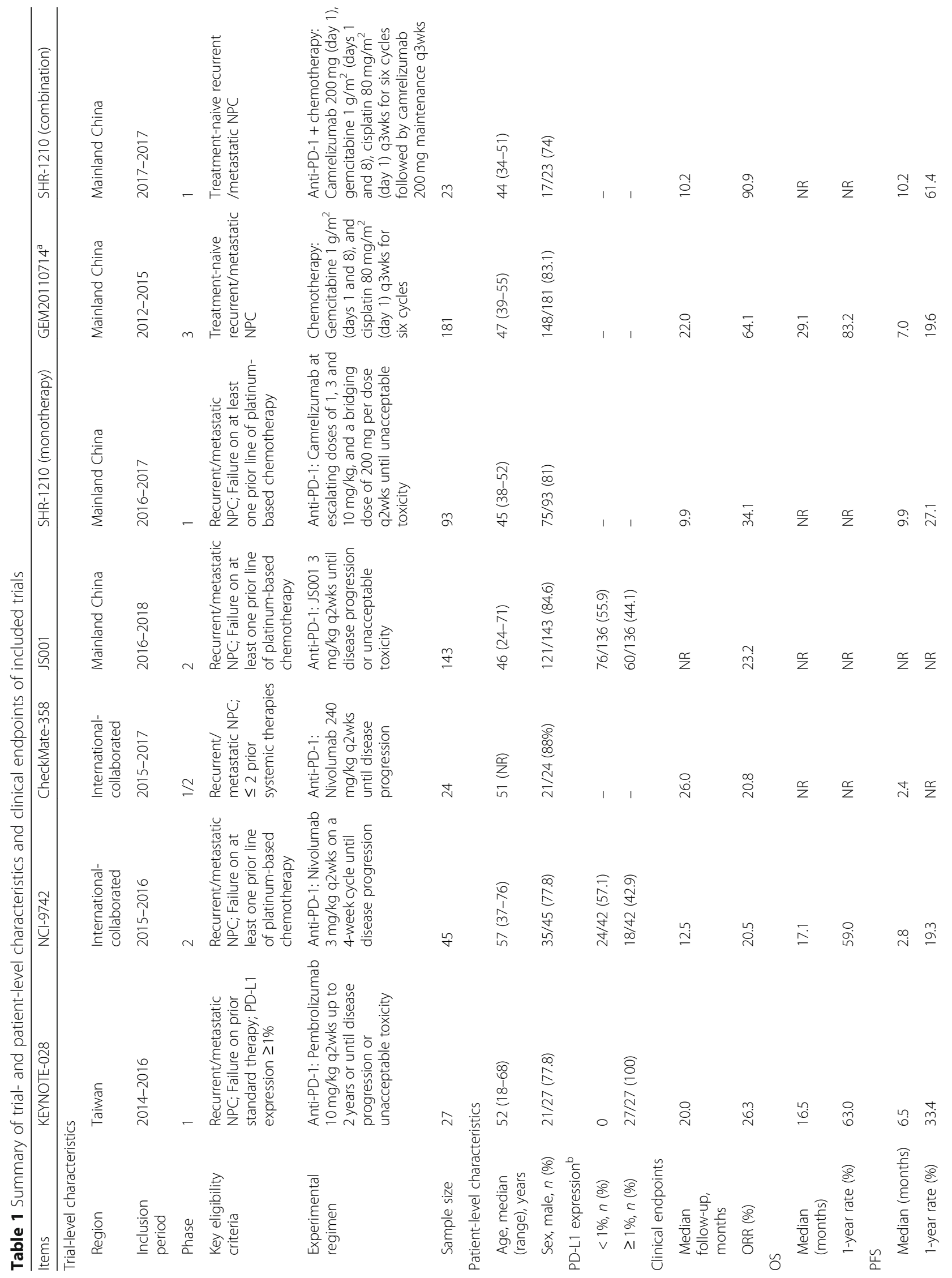




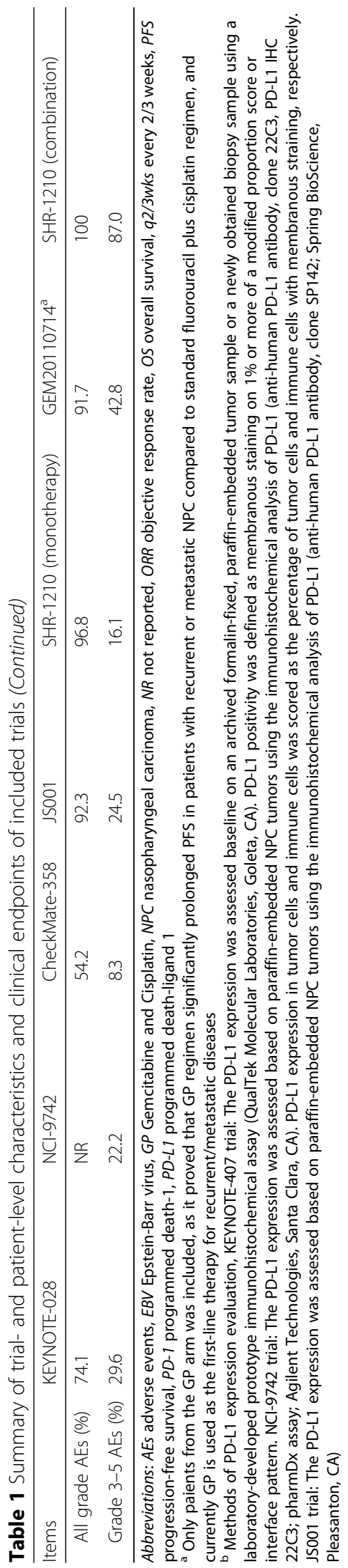

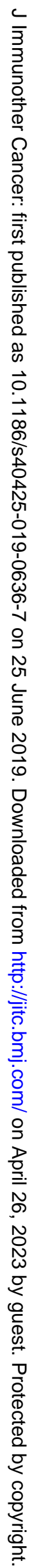




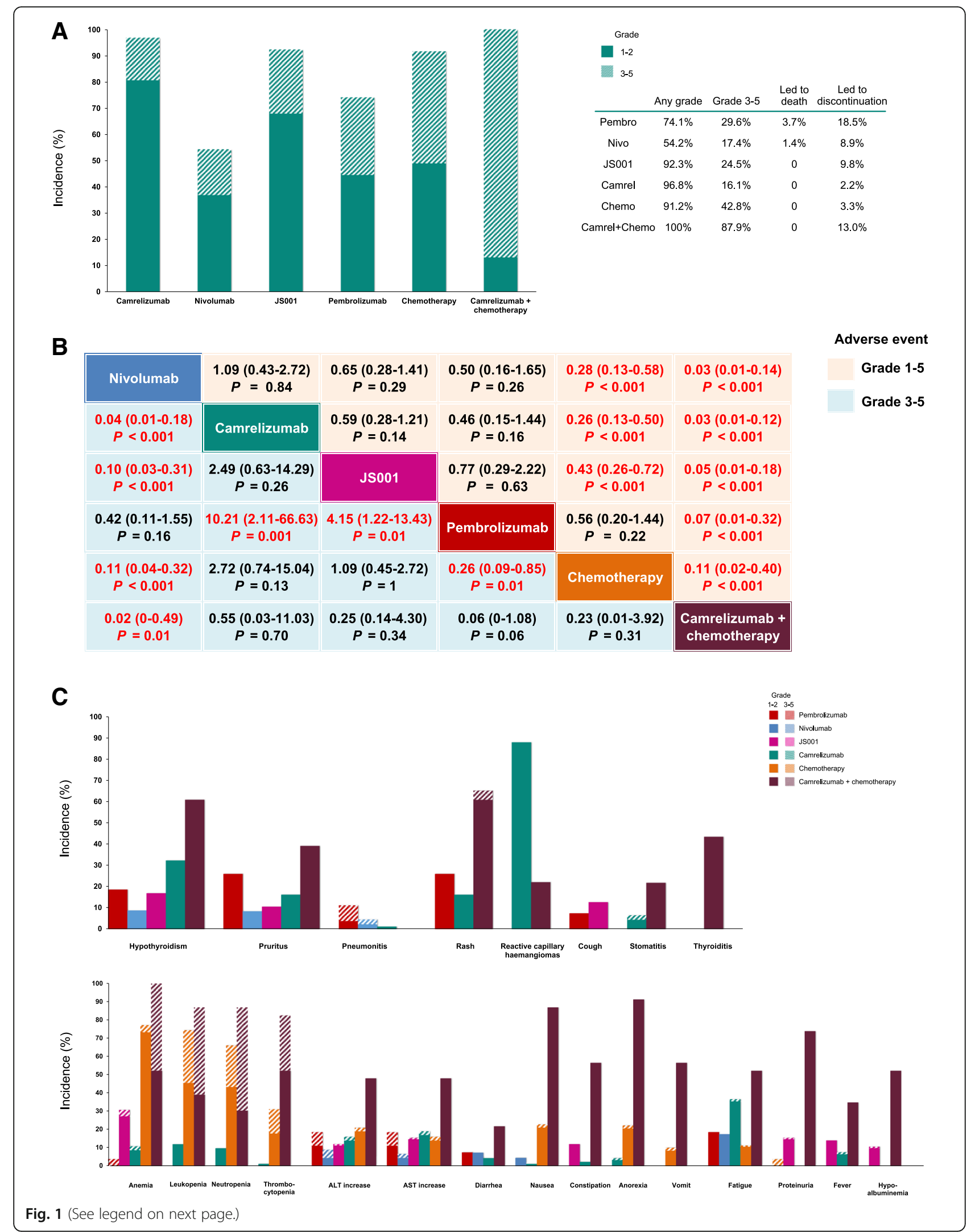


(See figure on previous page.)

Fig. 1 Safety profiles of anti-PD-1 monotherapy, chemotherapy alone, or their combination in advanced nasopharyngeal carcinoma. a Bar plot depicts the incidence rates of grade 1-5 adverse events (divided into grade 1-2 and 3-5) in pembrolizumab, nivolumab, JS001, camrelizumab, chemotherapy, and camrelizumab plus chemotherapy. The rates of deaths and discontinuation rates due to adverse events are also presented. $\mathbf{b}$ Indirect comparisons of grade $1-5$ and 3-5 adverse events in different regimens. The pooled odds ratios and $95 \%$ confidence intervals indicate the result of the top regimen versus the bottom regimen. Each cell contains the pooled odds ratios and $95 \%$ confidence intervals; significant results are indicated in red. c Bar plot depicts the toxicity spectra based on each of the specific adverse event. The upper section shows the incidence rates of immune-related adverse events and the lower section shows the incidence rate of other common class-specific adverse events. The incidence rates of pneumonitis in camrelizumab plus chemotherapy, and thyroiditis, proteinuria, hypoalbuminemia and vomit in camrelizumab were reported to be zero. The grade 1-2 adverse events of anemia and proteinuria were not reported for pembrolizumab

and other class-specific common AEs (Fig. 1c). Immunerelated AEs among different anti-PD-1 drugs included hypothyroidism (range, $8.7-32.3 \%$ ), pruritus (8.3-16.1\%), and rash (16.1-25.9\%); camrelizumab was reported to have a notably high incidence of reactive capillary haemangiomas (88.0\%). Majority of immune-related AEs were mild and moderate (grade 1-2). Grade 3-5 immunerelated AEs included pneumonitis (7.4\% in pembrolizumab and $2.2 \%$ in nivolumab), rash $(4.3 \%$ in camrelizumab plus chemotherapy), and stomatitis (2.2\% in camrezumab alone) (Fig. 1c). In terms of other common AEs, elevated alanine aminotransferase or aspartate aminotransferase, fatigue, and anemia were observed (Fig. 1c). Of note, the incidence of immune-related and other common AEs increased substantially in camrelizumab+chemotherapy, compared to camrelizumab or chemotherapy alone: common grade 1-5 AEs included anemia (100\%; grade $3-5,47.8 \%)$, anorexia $(91.3 \%)$, neutropenia $(87.0 \%$; grade $3-5,56.6 \%)$, leukopenia $(87.0 \%$; grade $3-5,47.8 \%)$, nausea $(87.0 \%)$, thrombocytopenia (82.6\%; grade $3-5,30.4 \%)$, proteinuria $(73.9 \%)$, rash (65.2\%; grade $3-5,4.3 \%)$, hypothyroidism $(60.9 \%)$; the incidences of thyroiditis (43.5\%) and pruritus (39.1\%) were also relatively higher (Fig. 1c).

\section{Efficacy of different regimens}

Figure 2a presents the efficacy of different regimens. The ORR of anti-PD-1 monotherapy used as $>1$ st line therapy ranged $19.0-34.1 \%$, relatively higher in camrelizumab (34.1\%), followed by pembrolizumab (26.3\%), JS001 (23.3\%), and nivolumab (19.0\%). Intriguingly, when nivolumab was used as first-line therapy, its ORR increased to $40.0 \%$ (Fig. 2a). Camrelizumab+chemotherapy combination treatment in first-line therapy dramatically increased the ORR from $64.1 \%$ (chemotherapy alone) to 90.9\% (Fig. 2a). It was noteworthy that similar complete response (CR) rates between GP chemotherapy (8.3\%) and anti-PD- $1+$ chemotherapy (4.5\%) were observed, though the partial response (PR) rate of anti-PD-1+ chemotherapy (86.4\%) was substantially higher than that of GP chemotherapy (55.8\%). Pooled ORR for PD-L1positive patients was $28.4 \%$ versus $17.4 \%$ of those with PD-L1-negative tumors $(P=0.11)$ (Fig. 2b).

\section{Discussion}

This is the first report that compares the safety and efficacy of different anti-PD-1 drugs with/without chemotherapy in RM-NPC, which provides preliminary evidence and integrative insights into the future design and implementation of immune clinical trials in NPC. The general safety of nivolumab and pembrolizumab ranked high, while the incidences of grade 3-5 AEs were relatively low in camrelizumab and nivolumab. Integrating with the specific toxicity spectra of each drug, we postulate that camrelizumab has the least toxicity profile; the high incidence of all-grade AEs may be attributed to reactive capillary hemangiomas, which is generally unthreatening and self-resolved [8]. In general, anti-PD-1 therapy was safer than standard chemotherapy; however, once it was combined with chemotherapy, the incidence of grade 3-5 AEs and AE-related discontinue rate doubled over chemotherapy alone, which suggests potentially synergized toxicity. This is in accordance with the safety profiles in the KEYNOTE-048 trial investigating anti-PD$1+$ chemotherapy versus chemotherapy alone in nonnasopharynx head and neck cancer [11].

The response rate with anti-PD-1 monotherapy for pretreated RM-NPC approximated 20-30\%, whereas ORR increased to $40 \%$ in treatment-naive patients. Additionally, the incidences of AEs with anti-PD-1 regimen were generally lower than those with chemotherapy. Though the sample size in first-line anti-PD-1 group is limited, it provides preliminary evidences that comparing first-line anti-PD-1 monotherapy versus standard chemotherapy is a "trial-worthy" approach.

Interestingly, we observed similar $\mathrm{CR}$ rates between GP chemotherapy and anti-PD- $1+$ chemotherapy. Considering that anti-PD-1 monotherapy only achieved limited $C R$ rate $(\sim 0-2 \%)$, it might suggest that the addition of anti-PD-1 agents may not be adequate enough to increase complete elimination of tumor cells (CR rate), though this combination therapy can still substantially improve tumor killing efficacy (PR rate). Likewise, a phase 2 trial by Chia and colleagues [12] reported a similar CR rate $(8.6 \%)$ and a relatively higher PR rate $(62.9 \%)$ in treatment-naive RM-NPC receiving chemotherapy followed by EBV-specific cytotoxic T lymphocytes (CTLs), compared to that of GP chemotherapy 

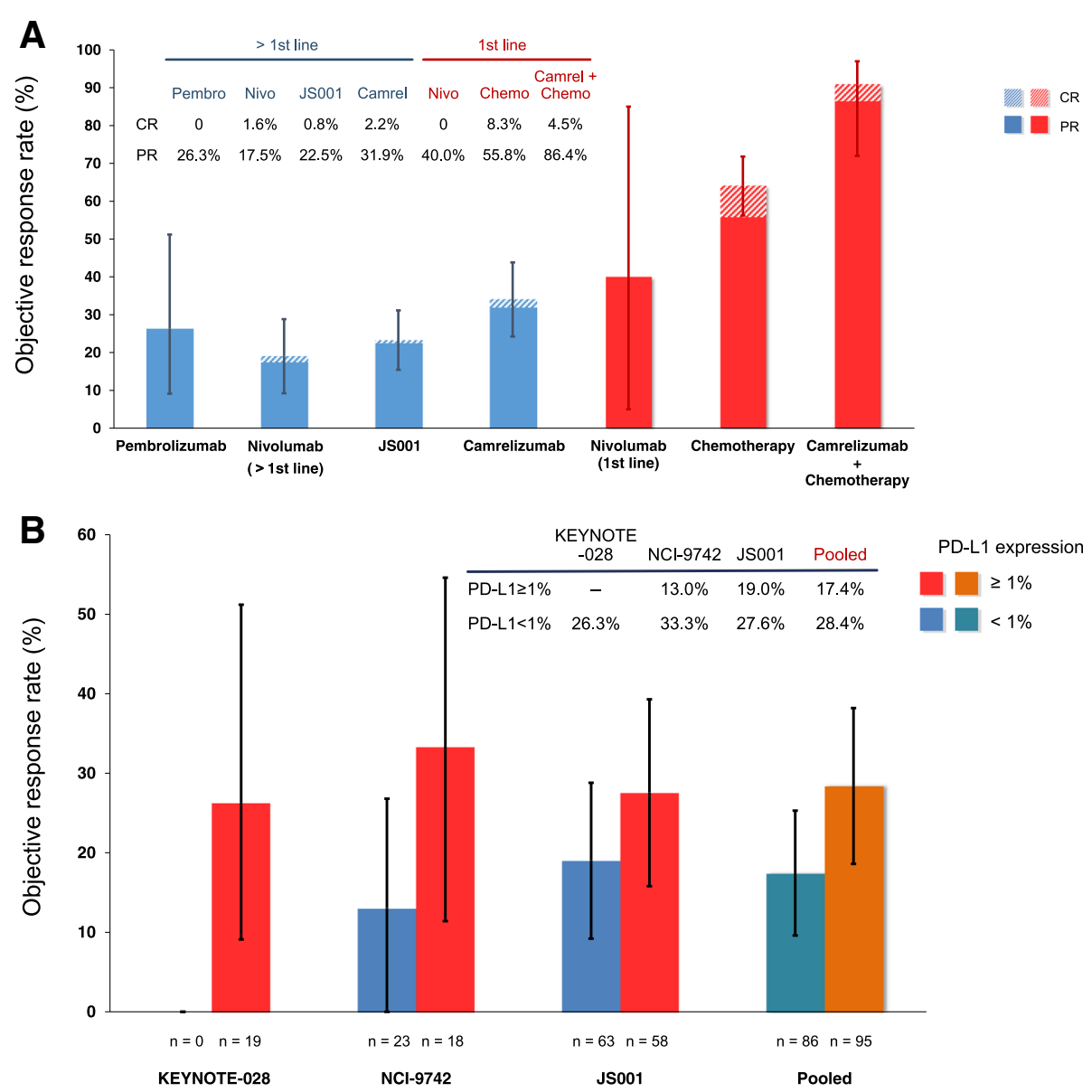

Fig. 2 Efficacy of anti-PD-1 monotherapy, chemotherapy alone, or their combination therapy in advanced nasopharyngeal carcinoma. a Bar plot shows the proportion of patients with response to pembrolizumab, nivolumab (first-line and > 1st line), JS001, camrelizumab, chemotherapy, and camrelizumab plus chemotherapy. $\mathbf{b}$ Bar plot depicts objective response rates of anti-PD-1 therapies according to the level of programmed deathligand 1 (PD-L1) expression; data were available from the three trials (KEYNOTE-028, NCl-9742, and JS001) and their pooled analysis. KEYNOTE-028 trial only enrolled patients with PD-L1-positive tumors. Error bars reflect 95\% confidence intervals of objective response rates. CR $=$ complete response, $\mathrm{PR}=$ partial response

(Table 2). These results implicate that the combination of immunotherapy agents (either anti-PD-1 or EBVCTLs) with chemotherapy may not achieve synergic effects (similar CR rates) in NPC patients; however, the substantially increased $\mathrm{PR}$ rate may still translate into patient survival benefits. Our findings need to be verified in ongoing/future prospective randomized trials evaluating the combination of immunotherapy and chemotherapy versus chemotherapy alone.

One major challenge of immunotherapy remains that it only benefits small subsets of patients. Prior trial data $[6,9]$ showed a numerically higher ORR in patients with PD-L1-positive RM-NPC than in those with PD-L1negative tumors; and high-PD-L1 expression was associated with better survival outcomes in both NPC and other head and neck cancer [13, 14]. We further performed pooled analysis to increase statistical power. Unfortunately, a significant margin was still not reached; the possible reason would be limited sample size. Moreover, PD-L1 expression alone may not be the only determinant of treatment benefits; it might be contingent on other factors in the tumor microenvironment that are yet to be identified. Future studies are warranted to identify reliable biomarkers for tailoring anti-PD-1 therapies. Improvement in treatment efficacy can also be achieved by breakthroughs in combination therapy. It is shown that camrelizumab+GP combination approach achieved a remarkable ORR in first-line treatment. Additionally, previous exposure to ipilimumab significantly improved antitumor activity of camrelizumab [8]. Going forward, these preliminary findings construct a roadmap for the design of future trials to assess the efficacy of immuno-oncology cocktail and/or dual inhibition of immune checkpoints approaches in NPC. Results from relevant ongoing trials (e.g. NCT03581786, NCT03707509, NCT03097939) are eagerly awaited. 
Table 2 Summary of characteristics and clinical endpoints of the Singapore trial evaluating the combination of chemotherapy and adoptive T-cell therapy in NPC

\begin{tabular}{ll}
\hline Items & Singapore trial \\
\hline $\begin{array}{l}\text { Characteristics } \\
\text { Phase }\end{array}$ & 2 \\
$\begin{array}{l}\text { Key eligibility criteria } \\
\text { Experimental }\end{array}$ & Treatment-naïve recurrent/metastatic EBV-positive NPC \\
regimen & Chemotherapy+EBV-CTLs: Gemcitabine $1000 \mathrm{mg} / \mathrm{m}^{2}$, and carboplatin (AUC 2) (days 1, 8 and 15) q4wks for 4 cycles, followed \\
$\begin{array}{l}\text { Sample size } \\
\text { Clinical endpoints }\end{array}$ & 35 \\
Median follow-up, & 29.9 \\
months & \\
ORR (\%) & 71.5 \\
CR (\%) & 8.6 \\
PR (\%) & 62.9 \\
SD (\%) & 28.6 \\
PD (\%) & 0 \\
All grade AEs (\%) & NR \\
Grade 3-5 AEs (\%) & NR
\end{tabular}

Abbreviations: $A U C$ area under the curve, $C R$ complete response, EBV Epstein-Barr virus, EBV-CTLS EBV-specific cytotoxic T lymphocytes, NPC nasopharyngeal carcinoma, NR not reported, ORR objective response rate, $q 4 w k s$ every 4 weeks, $P D$ progressive disease, $P R$ partial response, $S D$ stable disease

One major limitation of this study is that all trials were in phase $1 / 2$, therefore long-term survival data are still lacking and the sample sizes were limited, especially for first-line nivolumab $(n=5)$ and the combination therapy group $(n=23)$. Our findings need to be verified in future large-scale, head-to-head, phase 3 trials. Secondly, the results of subgroup analyses regarding PD-L1 expression level should be interpreted with cautions, in view of the different immunohistochemical assays used $[5,6,9]$.

\section{Conclusions}

Our study comprehensively compares the safety profile and efficacy of anti-PD-1 monotherapy, chemotherapy and their combination in RM-NPC, which provides important evidence for the design of future trials and clinical management with respect to anti-PD-1 therapy.

\section{Abbreviations}

AE: Adverse event; Cl: Confidence interval; CR: Complete response; CTL: Cytotoxic T lymphocytes; ESMO: European Society for Medical Oncology; GP: Gemcitabine plus cisplatin; NPC: Nasopharyngeal carcinoma; OR: Odds ratio; ORR: Objective response rate; PD-L1: Programmed deathligand 1; PR: Partial response; RM-NPC: Recurrent or metastatic NPC

\section{Acknowledgements}

Not applicable.

\section{Authors' contributions}

$Y C, J L$, and ZW concept and design this study. $Y C, J L$, and ZW make the data and statistical analysis. All authors write the manuscript. All authors read and approved the final manuscript.

\section{Funding}

This work was supported by grants from the National Natural Science Foundation of China (No. 81802707).

\section{Availability of data and materials}

The datasets used and/or analyzed during the current study are available from the corresponding author on reasonable request.

Ethics approval and consent to participate

Not applicable.

Consent for publication

Not applicable.

\section{Competing interests}

The authors declare that they have no competing interests.

Received: 2 January 2019 Accepted: 12 June 2019

Published online: 25 June 2019

\section{References}

1. Torre LA, Bray F, Siegel RL, Ferlay J, Lortet-Tieulent J, Jemal A. Global cancer statistics, 2012. CA Cancer J Clin. 2015;65(2):87-108.

2. Zhang L, Huang Y, Hong S, Yang Y, Yu G, Jia J, et al. Gemcitabine plus cisplatin versus fluorouracil plus cisplatin in recurrent or metastatic nasopharyngeal carcinoma: a multicentre, randomised, open-label, phase 3 trial. Lancet. 2016;388(10054):1883-92.

3. Fang W, Zhang J, Hong S, Zhan J, Chen N, Qin T, et al. EBV-driven LMP1 and IFN-gamma up-regulate PD-L1 in nasopharyngeal carcinoma: implications for oncotargeted therapy. Oncotarget. 2014;5(23):12189-202.

4. Wang YQ, Chen YP, Zhang Y, Jiang W, Liu N, Yun JP, et al. Prognostic significance of tumor-infiltrating lymphocytes in nondisseminated nasopharyngeal carcinoma: a large-scale cohort study. Int J Cancer. 2018; 142(12):2558-66.

5. Hsu C, Lee SH, Ejadi S, Even C, Cohen RB, Le Tourneau C, et al. Safety and antitumor activity of Pembrolizumab in patients with programmed deathligand 1-positive nasopharyngeal carcinoma: results of the KEYNOTE-028 study. J Clin Oncol. 2017;35(36):4050-6. 
6. Ma BBY, Lim WT, Goh BC, Hui EP, Lo KW, Pettinger A, et al. Antitumor activity of Nivolumab in recurrent and metastatic nasopharyngeal carcinoma: an international, multicenter study of the Mayo Clinic phase 2 consortium (NCl-9742). J Clin Oncol. 2018:36(14):1412-8.

7. Delord JP, Hollebecque A, De Boer JP, De Greve J, Machiels JPH, Leidner RS, et al. An open-label, multicohort, phase I/ll study to evaluate nivolumab in patients with virus-associated tumors (CheckMate 358): efficacy and safety in recurrent or metastatic (R/M) nasopharyngeal carcinoma (NPC). J Clin Oncol. 2017;35(15_suppl):6025.

8. Fang W, Yang Y, Ma Y, Hong S, Lin L, He X, et al. Camrelizumab (SHR-1210) alone or in combination with gemcitabine plus cisplatin for nasopharyngeal carcinoma: results from two single-arm, phase 1 trials. Lancet Oncol. 2018; 19(10):1338-50.

9. Xu R, Wang FH, Feng FJ, Li Q, Xu N, Hu XC, et al. 1150PRecombinant humanized anti-PD-1 monoclonal antibody (JS001) in patients with refractory/metastatic nasopharyngeal carcinoma: Preliminary results of an open-label phase II clinical study. Ann Oncol. 2018;29(suppl_8):mdy288.023.

10. Lawson R. Small sample confidence intervals for the odds ratio. Commun Stat - Simul Comput. 2004;33(4):1095-113.

11. Burtness B, Harrington KJ, Greil R, Soulières D, Tahara M, De Castro JG, et al. LBA8_PRKEYNOTE-048: phase III study of first-line pembrolizumab (P) for recurrent/metastatic head and neck squamous cell carcinoma (R/M HNSCC). Ann Oncol. 2018;29(suppl_8):mdy424.045.

12. Chia WK, Teo M, Wang WW, Lee B, Ang SF, Tai WM, et al. Adoptive T-cell transfer and chemotherapy in the first-line treatment of metastatic and/or locally recurrent nasopharyngeal carcinoma. Mol Ther. 2014;22(1):132-9.

13. Zhu Q, Cai MY, Chen CL, Hu H, Lin HX, Li M, et al. Tumor cells PD-L1 expression as a favorable prognosis factor in nasopharyngeal carcinoma patients with pre-existing intratumor-infiltrating lymphocytes. Oncoimmunology. 2017;6(5):e1312240.

14. Jayaraj R, Kumarasamy C, Samiappan S, Swaminathan P. Letter to the editor regarding, "the prognostic role of PD-L1 expression for survival in head and neck squamous cell carcinoma: a systematic review and meta-analysis". Oral Oncol. 2019;90:139-40.

\section{Publisher's Note}

Springer Nature remains neutral with regard to jurisdictional claims in published maps and institutional affiliations.

Ready to submit your research? Choose BMC and benefit from:

- fast, convenient online submission

- thorough peer review by experienced researchers in your field

- rapid publication on acceptance

- support for research data, including large and complex data types

- gold Open Access which fosters wider collaboration and increased citations

- maximum visibility for your research: over $100 \mathrm{M}$ website views per year

At $\mathrm{BMC}$, research is always in progress.

Learn more biomedcentral.com/submissions 